\title{
View-Based Maps
}

\author{
Kurt Konolige, James Bowman, JD Chen, Patrick Mihelich Michael Calonder, Vincent Lepetit, Pascal Fua \\ Willow Garage \\ Menlo Park, CA 94025 \\ Email: konolige@willowgarage.com \\ EPFL \\ Lausanne, Switzerland \\ Email: michael.calonder@epfl.ch
}

\begin{abstract}
Robotic systems that can create and use visual maps in realtime have obvious advantages in many applications, from automatic driving to mobile manipulation in the home. In this paper we describe a mapping system based on retaining stereo views of the environment that are collected as the robot moves. Connections among the views are formed by consistent geometric matching of their features. Out-of-sequence matching is the key problem: how to find connections from the current view to other corresponding views in the map. Our approach uses a vocabulary tree to propose candidate views, and a strong geometric filter to eliminate false positives - essentially, the robot continually re-recognizes where it is. We present experiments showing the utility of the approach on video data, including map building in large indoor and outdoor environments, map building without localization, and re-localization when lost.
\end{abstract}

\section{INTRODUCTION}

Fast, precise, robust visual mapping is a desirable goal for many robotic systems, from transportation to in-home navigation and manipulation. Vision systems, with their large and detailed data streams, should be ideal for recovering 3D structure and guiding tasks such as manipulation of everyday objects, navigating in cluttered environments, and tracking and reacting to people. But the large amount of data, and its associated perspective geometry, also create challenging problems in organizing the data in an efficient and useful manner.

One useful idea for maintaining the spatial structure of visual data is to organize it into a set of representative views, along with spatial constraints among the views, called a skeleton. Figure 1 gives an example of a skeleton constructed in an indoor environment. Typically views are matched in sequence as the camera is moved around, so the skeleton mimics the camera trajectory (red trajectory). In loop closure, the camera enters an area already visited, and can re-connect with older views. The overall map is generated by nonlinear optimization of the system $[2,19,33]$. View-based maps have the advantage of scalability: using incremental techniques, new views can be added and the skeleton optimized online.

One problem is how to efficiently perform loop closure. Previous approaches used exhaustive search of the current view against all skeleton views that could possibly be in the area, given the relative uncertainty of views. This approach does not scale well to larger skeletons, and involves constant calculation of relative covariance. Instead, to limit the number of views that must be considered for loop closure, we employ a vocabulary tree [27] to suggest candidate views, a type

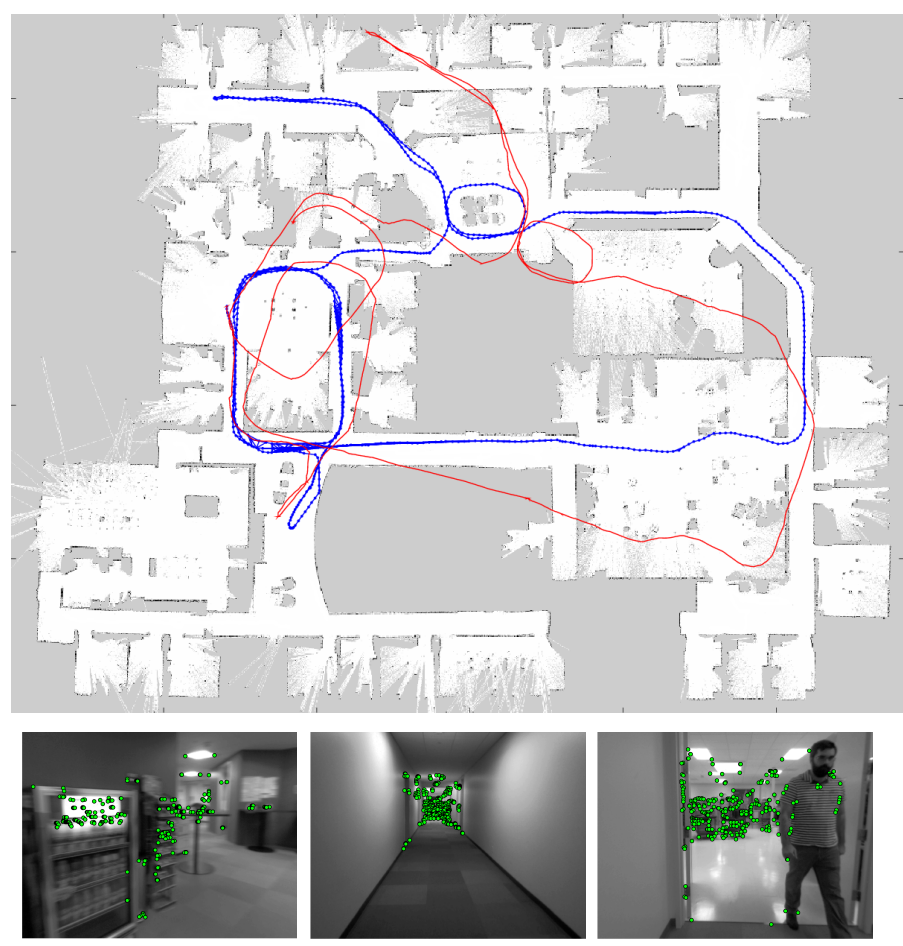

Fig. 1. Top: Skeleton map constructed online from just stereo images, registered against a laser map for reference. Red is visual odometry, blue is corrected by loop closure from visual place recognition. Tick marks at sides of map are $10 \mathrm{~m}$ intervals. Bottom shows typical views, with blurring, clutter, people, and blank walls.

of place recognition (PR). The vocabulary tree allows us to efficiently filter thousands of skeleton views to find possible matches, as well as add new views online. We call this online PR re-recognition: the robot recognizes its position relative to the stored view map on every cycle, without any a priori knowledge of its position (unlike localization, which requires a position hypothesis).

The addition of vocabulary tree PR to view-based maps is a happy alignment of technologies that expands the utility of visual mapping in interesting ways. For example, even without sequence information, it is often possible to quickly reconstruct a skeleton map from a set of views (Figure 9 and Section VI-D). In the Experiments section, we highlight some other applications that show the scalability of viewbased maps. Loop closure over large distances is possible: we show indoor maps with $800 \mathrm{~m}$ trajectories (Figure 1), and outdoor rough-terrain maps with $5 \mathrm{~km}$ trajectories. On a smaller scale, view matching with large numbers of points is inherently 
accurate, showing a few centimeter accuracy over a desktop workspace. Additional capabilities include automatic recovery from localization failures (e.g., occlusion and motion blur) and incremental construction of maps.

The main contributions of this paper are

- The construction of a realtime system for robust, accurate visual map making over large and small spaces.

- The use of views (images), view matching, and geometric relations between views as a uniform approach to shortterm tracking and longer-term metric mapping and loop closure.

- The integration of a visual vocabulary tree into a complete solution for online place recognition.

- An analysis of the false positive rejection ability of twoview geometry.

- Extensive experiments with real data, showing the scalability of the technique.

Our solution uses stereo cameras for input images. The development of place recognition is also valid for monocular cameras, with the exception that the geometric check is slightly stronger for stereo. However, the skeleton system so far has been developed just for the full 6DOF pose information generated by stereo matching, and although it should be possible to weaken this assumption, we have not yet done so.

\section{VSLAM AND VIEW MAPS}

The view map system (Figure 2), which derives from FrameSLAM [2, 23], is most simply explained as a set of nonlinear constraints among camera views, represented as nodes and edges (see Figure 5 for a sample graph). Constraints are input to the graph from two processes, visual odometry (VO) and place recognition (PR). Both rely on geometric matching of views to find relative pose relationships; they differ only in their search method. VO continuously matches the current frame of the video stream against the last keyframe, until a given distance has transpired or the match becomes too weak. This produces a stream of keyframes at a spaced distance, which become the backbone of the constraint graph, or skeleton. PR functions opportunistically, trying to find any other views that match the current keyframe. This is much more difficult, especially in systems with large loops. Finally, an optimization process finds the best placement of the nodes in the skeleton.

It is interesting to note that current methods in visual SLAM divide in the same way as in laser-based SLAM, namely, those that keep track of landmarks using an EKF filter (monoSLAM [9, 10] and variations [29, 32]), and those that, like ours, maintain a constraint graph of views, similar to the original $\mathrm{Lu}$ and Milios method [25]. The main limitation of the landmark methods is the filter size, which is only tractable in small (room-size) environments. An exception is [29], which uses a submap technique, although realtime performance has not yet been demonstrated. Landmark systems also tend to be less accurate, because they typically track only a few tens of landmarks per frame. In contrast, our visual odometry technique tracks 300 points per frame, and we construct

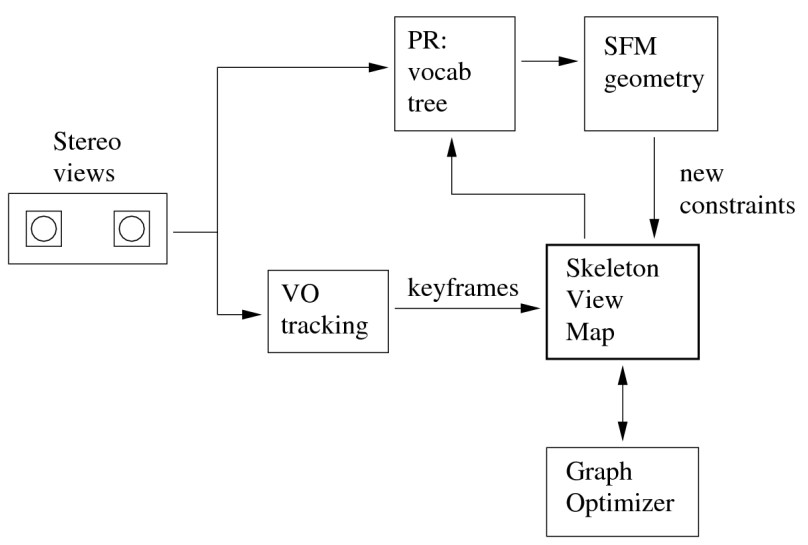

Fig. 2. System overview.

maps containing several thousand views (and thus hundreds of thousands of points).

In a similar vein, the recent Parallel Tracking and Mapping (PTAM) system [20, 21] also uses 3D landmarks, but employs standard SfM bundle adjustment to build a map from many views. Many more points can be handled in the decoupled tracking phase, leading to accurate and robust performance under many conditions. Still, it is limited to small environments (around 150 keyframes) by the number of points and by bundle adjustment. It is also subject to tracking failures on self-similar textures (e.g., bushes), object motion, and scene changes (e.g., removal of an object). In contrast, view-based maps use consistent view geometry to robustly estimate poses even in the presence of distractors.

The skeleton system deployed here comes directly from the frameSLAM work in [2, 23]. Several other systems employ constraint graphs as the basic map structure. Fraundorfer et al. [13] have a monocular system that represents only direction information between views, and produce only a topological map. Eade and Drummond [11] employ a hybrid approach, using EKF landmarks within a local area called a node, then connecting the nodes via similarity relations. An interesting point of their graph optimization is the use of cycles to constrain relative scale between nodes. Other robotics work that employs similar ideas about constructing view-based constraints is in [33, 34]. These systems also keep a constraint network of relative pose information between frames, based on stereo visual odometry, and solve it using nonlinear least square methods. The main difference with our system is that frameSLAM represents the relationships as nonlinear constraints, which are more accurate over angular deformations, and can reduce the size of the skeleton graph to deal with larger areas as required.

\section{Related Place Recognition Work}

Visual place recognition is an image classification problem; new views are classified against a set of previously seen views. For use in VSLAM, the classifier must support efficient online learning of new reference views. Image matching techniques based on bag-of-words matching are ideally suited to this purpose. For fast lookup of similar places, we rely 
on the hierarchical vocabulary trees proposed by Nistér and Stewénius [27], which has the advantage of fast online learning of new places. Other methods include alternative approximate nearest neighbor algorithms $[30,26]$ and various refinements for improving the response or efficiency of the tree $[8,17,18]$.

Cummins and Newman [8] show how to use visual features for navigation and loop closure over very large trajectories. They use pairwise feature statistics and sequences of views to address the perceptual aliasing problem, especially notable in man-made environments containing repeated structure. Jegou et al. [17] incorporate Hamming embedding and weak geometric consistency constraints into the inverted file to improve performance. In this work, we rely instead on a strong geometric consistency check on single views.

Jegou et al. [18] note that even using inverted files, query time is linear in the number of reference images; they propose a two-level inverted file scheme to improve the complexity. Our experiments do show linearly increasing query/update time, but with a very small constant factor (Figure 6). For our scale of application (in the thousands of images), the query time of the vocabulary tree is nearly constant, and such sophistication is unnecessary.

In application to graph-based VSLAM, Callmer et al. [4] propose a loop closure procedure that uses a vocabulary tree in a manner similar to ours, along with a weak geometric check to weed out some false positives. Eade and Drummond [12] have extended their node approach with a PR method based on bag of words, in which they learn the words online. They give few statistics on the performance of PR, so it isn't possible to compare directly - they have the advantage of learning based on the observed features, but have far fewer words (3000 vs. 100,000 in our case). They have independently introduced some of the same applications of PR as given here: recovery from localization error and stitching together trajectories when common views are found. Finally, Williams et al. [35] also recover from localization errors in a landmarkbased VSLAM framework, by training a classifier to recognize landmarks online; so far their system has been limited to 80 landmarks, mostly because of EKF processing.

There is an interesting convergence between our work and recent photo stitching in the vision community [31]. They employ a similar skeletonization technique to limit the extent of bundle adjustment calculations, but run in batch mode, with no attempt at realtime behavior. Klopschitz et al. [22] use a vocabulary tree to identify possible matches in video stream, and then followed by a dynamic programming technique to verify a sequence of view matches. They are similar to our work in emphasizing online operation.

\section{FRAMESLAM BACKGROUND}

The skeleton view map encodes 6DOF relations bewteen views. For two views $c_{i}$ and $c_{j}$ with a known relative pose, the constraint between them is

$$
\Delta z_{i j}=c_{i} \ominus c_{j}, \text { with covariance } \Lambda^{-1}
$$

where $\ominus$ is the inverse motion composition operator - in other words, $c_{j}$ 's position in $c_{i}$ 's frame. The covariance expresses the strength of the constraint, and arises from the geometric matching step that generates the constraint, explained below.

Given a constraint graph, the optimal position of the nodes is a nonlinear optimization problem of minimizing $\sum_{i j} \Delta z_{i j}^{\top} \Lambda \Delta z_{i j}$; a standard solution is to use preconditioned conjugate gradient $[2,16]$. For realtime operation, it is more convenient to run an incremental relaxation step, and the recent work of Grisetti et al. [15] on SGD provides an efficient method of this kind, called Toro, which we use for the experiments. This method selects a subgraph to optimize, and runs only a few iterations on each addition to the graph. Other relaxation methods for nonlinear constraint systems include $[14,28]$.

\section{A. Geometric Consistency Check and Pose Estimation}

Constraints arise from the perspective view geometry between two stereo camera views. The process can be summarized by the following steps:

1) Match features in the left image of one view with features in the left image of the other view $(N \times N$ matching).

2) (RANSAC steps) From the set of matches, pick three candidates, and generate a relative motion hypothesis between the views. Stereo information is essential here for giving the 3D coordinates of the points.

3) Project the $3 \mathrm{D}$ points from one view onto the other based on the motion hypothesis, and count the number of inliers.

4) Repeat 2 and 3, keeping the hypothesis with the best number of inliers.

5) Polish the result by doing nonlinear estimation of the relative pose from all the inliers.

The last step iteratively solves a linear equation of the form

$$
J^{\top} J \delta x=-J^{\top} \Delta z
$$

where $\Delta z$ is the error in the projected points, $\delta x$ is a change in the relative pose of the cameras, and $J$ is the Jacobian of $z$ with respect to $x$. The inverse covariance derives from $J^{\top} J$, which approximates the curvature at the solution point. As a practical matter, Toro accepts only diagonal covariances, so instead of using $J^{\top} J$, we scale a simple diagonal covariance based on the inlier response.

In cases where there are too few inliers, the match is rejected; this issue is explored in detail in Section V-C. The important result is that geometric matching provides an almost foolproof method for rejecting bad view matches.

\section{B. Visual Odometry and Re-detection}

Our overriding concern is to make the whole system robust. In outdoor rough terrain, geometric view matching for VO has proven to be extremely stable even under very large image motion [24], because points are re-detected and matched over large areas of the image for each frame. For this paper's experiments, we use a recently-developed scale-space detector 
called STAR (similar to the CenSurE detector [1]) outdoors, and the FAST detector indoors. There is no motion assumption to drive keypoint match prediction - all keypoints are redetected at each frame. For each keypoint in the current left image, we search a corresponding area of size $128 \times 64$ pixels for keypoints in the reference keyframe image for a match using SAD correlation of a $16 \times 16$ patch. Robust geometric matching then determines the best pose estimate. Keyframes are switched when the match inlier count goes below 100, or the camera has moved $0.3 \mathrm{~m}$ or 10 degrees.

In a $400 \mathrm{~m}$ circuit of our labs, with almost blank walls, moving people, and blurred images on fast turns, there was not a single VO frame match failure (see Figure 5 for sample frames). The PTAM methods of [20], which employ hundreds of points per frame, can also have good performance, with pyramid techniques to determine large motions. However, they are prone to fail when there is significant object motion, since they do not explore the space of geometrically consistent data associations

\section{Skeleton Graph Construction}

The VO module provides a constant stream of keyframes to be integrated into the skeleton graph. To control the size of the graph for large environments, only a subset of the keyframes need to be kept in the graph. For example, in the $5 \mathrm{~km}$ outdoor runs, a typical distance between skeleton views is $5 \mathrm{~m}$.

As each keyframe is generated by VO, it is kept in a small sequential buffer until enough distance has accumulated to integrate it into the skeleton. At this point, all the views in the buffer are reduced to a single constraint between the first and last views in the buffer. The reduction process is detailed in [2]; for a linear sequence of constraints, it amounts to compounding the pose differences $\Delta z_{01} \oplus \Delta z_{12} \oplus \cdots \oplus$ $\Delta z_{n, n-1}$.

One can imagine many other schemes for skeleton construction that try to balance the density of the graph, but this simple one worked quite well. In the case of lingering in the same area for long periods of time, it would be necessary to stop adding new views to the graph, which otherwise would grow without limit. The frameSLAM graph reduction supports online node deletion, and we are starting to explore strategies for controlling the density of views in an area.

After incorporating a new view into the skeleton, the Toro optimizer is run for a few iterations to optimize the graph. The optimization can be amortized over time, allowing online operation for fairly large graphs, up to several thousand views (see the timings in Figure 6).

\section{Matching Views}

In this section we describe our approach to achieving efficient view matching against thousands of frames. We develop a filtering technique for matching a new image against a dataset of reference images (PR), using a vocabulary tree to suggest candidate views from large datasets. From a small set of the top candidates, we apply the geometric consistency check, using Randomized Tree signatures [5] as an efficient viewpoint-invariant descriptor for keypoint matching. Finally, we develop statistics to verify the rejection capability of this check.

\section{A. Compact Randomized Tree Signatures}

We use Randomized Tree (RT) signatures [5] as descriptors for keypoints. An RT classifier is trained offline to recognize a number of keypoints extracted from an image database, and all other keypoints are characterized in terms of their response to these classification trees. Remarkably, a fairly limited number of base keypoints-500 in our experiments-is sufficient. However, a limitation of this approach is that storing a pre-trained Randomized Tree takes a considerable amount of memory. A recent extension [6] compacts signatures into much denser and smaller vectors resulting in both a large decrease in storage requirement and substantially faster matching, at essentially the same recognition rates as RT signatures and other competing descriptors; Table I compares creation and matching times. The performance of compact signatures means that the $N \times N$ keypoint match of the geometric consistency check is not a bottleneck in the view matching process.

\begin{tabular}{c||c|c} 
& $\begin{array}{c}\text { Descriptor Creation } \\
(512 \mathrm{kpts})\end{array}$ & $\begin{array}{c}\mathrm{N} \times \mathrm{N} \text { Matching } \\
(512 \times 512 \mathrm{kpts})\end{array}$ \\
\hline Sparse RTs (CPU) & $31.3 \mathrm{~ms}$ & $27.7 \mathrm{~ms}$ \\
\hline Compact RTs (CPU) & $\mathbf{7 . 9} \mathbf{~ m s}$ & $\mathbf{6 . 3} \mathbf{~ m s}$ \\
\hline U-SURF64 (CPU) & $150 \mathrm{~ms}$ & $120 \mathrm{~ms}$ \\
& & $73 \mathrm{~ms}(\mathrm{ANN})$ \\
\hline U-SURF64 (GPU) & & $6.8 \mathrm{~ms}$
\end{tabular}

TABLE I

TIMINGS FOR DESCRIPTOR CREATION AND MATCHING.

\section{B. A Prefilter for Place Recognition}

We have implemented a place recognition scheme based on the vocabulary trees of Nistér and Stewénius [27] which has good performance for both inserting and retrieving images based on the compact RT descriptors. We call this step a prefilter because it just suggests candidates that could match the current view, which must then be subject to the geometric consistency check for confirmation and pose estimation. VO and PR both use the geometric check, but PR has the harder task of finding matches against all views in the skeleton, while VO only has to match against the reference keyframe. The prefilter is a bag-of-words technique that works with monocular views (the left image of the stereo pairs).

The vocabulary tree is a hierarchical structure that simultaneously defines both the visual words and a search procedure for finding the closest word to any given keypoint. The tree is constructed offline by hierarchical $k$-means clustering on a large training set of keypoint descriptors. The set of training descriptors is clustered into $k$ centers. Each center then becomes a new branch of the tree, and the subset of training descriptors closest to it are clustered again. The process repeats until the desired number of levels is reached.

The discriminative ability of the vocabulary tree increases with the number of words, at a cost of greater quantization 
error [3] and increased memory requirements. Nistér and Stewénius have shown that performance improves with the number of words, up to very large $(>1 \mathrm{M})$ vocabularies. In our experiments, we use about $1 \mathrm{M}$ training keypoints from 500 images in the Holidays dataset [17], with $k=10$, and create a tree of depth 5 , resulting in $100 \mathrm{~K}$ visual words. The Holidays dataset consists of mostly outdoor images, so the vocabulary tree is trained on data visually dissimilar to the indoor environments of most of our experiments.

The vocabulary tree is populated with the reference images by dropping each of their keypoint descriptors to a leaf and recording the image in a list, or inverted file, at the leaf. To query the tree, the keypoint descriptors of the query image are similarly dropped to leaf nodes, and potentially similar reference images retrieved from the union of the inverted files. In either case, the vocabulary tree describes the image as a vector of word frequencies determined by the paths taken by the descriptors through the tree. Each reference image is scored for relevance to the query image by computing the L1 distance between their frequency vectors. The score is entropyweighted to discount very common words using the Term Frequency Inverse Document Frequency (TF-IDF) approach described in $[27,30]$.

To evaluate the vocabulary tree as a prefilter, we constructed a small test set of some 180 keyframes over a $20 \mathrm{~m}$ trajectory, and determined ground truth matches by performing geometric matching across all $180 \times 180$ possibilities. In this dataset, each keyframe averages 11.8 ground truth matches. We inserted these keyframes, along with another 553 non-matching distractor keyframes, into the vocabulary tree. Querying the vocabulary tree with each of the 180 test keyframes in turn, we obtained their similarity scores against all the reference images. The sensitivity of the vocabulary tree matching is shown by the ROC curve (Figure 3, left) obtained by varying a threshold on the similarity score.

Since we can only afford to put a limited number of candidates through the geometric consistency check, the critical performance criterion is whether the correct matches appear among the most likely candidates. Varying $N$, we counted the percentage of the ground truth matches appearing in the top$N$ results from the vocabulary tree. For robustness, we want to be very likely to successfully relocalize from the current keyframe, so we also count the percentage of test keyframes with at least one or at least two ground truth matches in the top- $N$ results (Figure 3, right).

In our experiments, we take as match candidates the top $N=15$ responses from place recognition. We expect to find at least one good match for $97 \%$ of the keyframes and two good matches for $90 \%$ of the keyframes. For any given keyframe, we expect almost $60 \%$ of the correct matches to appear in the top 15 results.

\section{Geometric Consistency Check}

We can predict the ability of the geometric consistency check (Section IV-A) to reject false matches by making a few assumptions about the statistics of matched points, and
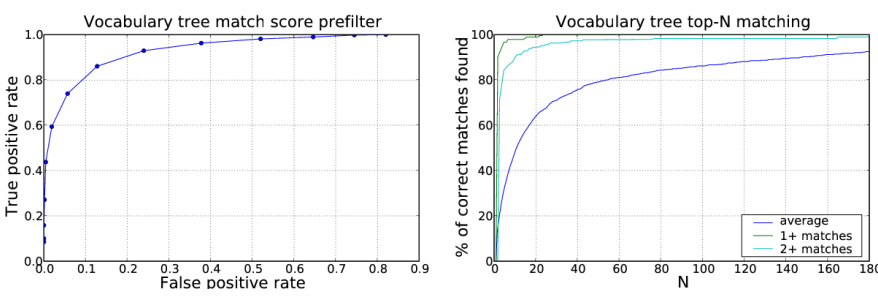

Fig. 3. Left: ROC curve for the vocabulary tree prefilter on the test dataset. Right: "Average" curve shows percentage of the correct matches among the top $N$ results from the vocabulary tree (blue); other curves are the percentage of views with at least 1 or 2 matches in the top $N$.

estimating the probability that two unrelated views $I_{0}$ and $I_{1}$ will share at least $M$ matches, given a relative pose estimate. Based on perspective geometry, any point match will be an inlier if the projection in $I_{1}$ lies on the epipolar line of the point in $I_{0}$. In our case, with $640 \times 480$ images, an inlier radius of 3 pixels, the probability of being an inlier is:

$$
A_{\text {track }} / A_{\text {image }}=(6 * 640) /(640 * 480)=.0125
$$

This is for monocular images; for stereo images, the two image disparity checks (assuming disparity search of 128 pixels) yield a further factor of $(6 / 128)^{*}(6 / 128)$. In the more common case with dominant planes, one of the image disparity checks can be ignored, and the factor is just (6/128). If the matches are random and independent (i.e., no common objects between images), then counting arguments can be applied. The distribution of inliers over $N$ trials with probability $p$ of being an inlier is $B_{p, N}$, the binomial distribution. We take the maximum inliers over $K$ RANSAC trials, so the probability of having less than $x$ inliers is $\left(1-B_{p, N}(x)\right)^{K}$. The probability of exactly $x$ inliers over all trials is

$$
\left(1-B_{p, N}(x)\right)^{K}-\left(1-B_{p, N}(x-1)\right)^{K}
$$

Figure 4 shows the probabilities for the planar stereo case, based on Equation 4. The graph peaks sharply at 2 inliers (out of 250 matches), showing the theoretic rejection ability of the geometric check. However, the real world has structure, and some keypoints form clusters: these factors violate the independent match assumption. Figure 4 compares actual rejections from the three datasets in the Experiments section, with two different types of keypoints, FAST and STAR. These show longer tails, especially FAST, which has very strong clustering at corners. Note that repetitive structure, which causes false positives for bag-of-words matching, as noted in [8], is rejected by the geometric check - for example, the windows in Figure 7. Even with the long tail, probabilities are very low for larger numbers of inliers, and the rejection filter can be set appropriately.

\section{EXPERIMENTS}

As explained in Section II, the view-based system consists of a robust $\mathrm{VO}$ detector that estimates incremental poses of a stereo video stream, and a view integrator that finds and adds non-sequential links to the skeleton graph, and optimizes the graph. We carried out a series of tests on stereo data from three different environments: 


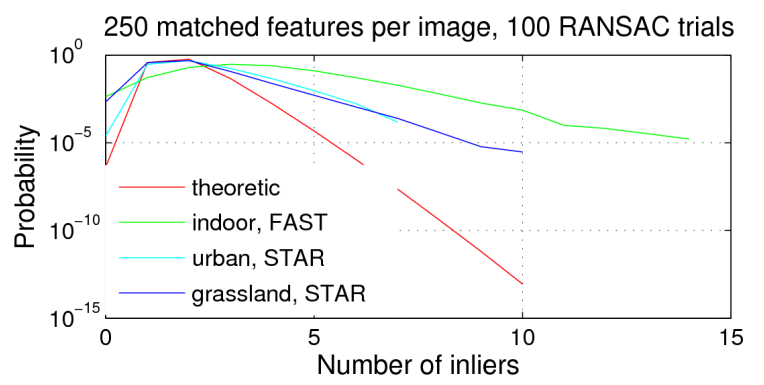

Fig. 4. The probability of getting $x$ inliers from a random unrelated view match. Theoretic probability (see text) compared to three different datasets. Note $\log$ scale for probabilities.

\begin{tabular}{c||c|c|c|c|c} 
Type & length & $\begin{array}{c}\text { image } \\
\text { res }\end{array}$ & $\begin{array}{c}\text { image } \\
\text { rate }\end{array}$ & $\begin{array}{c}\text { stereo } \\
\text { base }\end{array}$ & $\begin{array}{c}\text { skeleton } \\
\text { views }\end{array}$ \\
\hline Office & $0.8 \mathrm{~km}$ & $640 \times 480$ & $30 \mathrm{~Hz}$ & $9 \mathrm{~cm}$ & $4.2 \mathrm{k}$ \\
Urban & $0.4 \mathrm{~km}$ & $768 \times 568$ & $25 \mathrm{~Hz}$ & $100 \mathrm{~cm}$ & $0.5 \mathrm{k}$ \\
Terrain & $10 \mathrm{~km}$ & $512 \times 384$ & $15 \mathrm{~Hz}$ & $50 \mathrm{~cm}$ & $14.6 \mathrm{k}$
\end{tabular}

Rectification is not counted in timings; for the indoor sequence it is done in the stereo hardware. VO consumes $11 \mathrm{~ms}$ per video frame, leaving $22 \mathrm{~ms}$ for view integration, $2 / 3$ of the available time at the fastest frame rate. As in PTAM [20], view integration can be run in parallel with VO, so on a dualcore machine view matching and optimization could consume a whole processor. Given its efficiency, we publish results here for a single processor only. In all experiments, we restrict the number of features per image to $\sim 300$, and use 100 RANSAC iterations for geometric matching.

\section{A. Large Office Loop}

The first experiment is a large office loop of about $800 \mathrm{~m}$ in length. The trajectory was done by joysticking a robot at around $1 \mathrm{~m} / \mathrm{sec}$. Figure 1 shows some images: there is substantial blurring during fast turns, sections with almost blank walls, cluttered repetitive texture, and moving people. There are a total of $24 \mathrm{~K}$ images in the trajectory, with $10 \mathrm{k}$ keyframes, 4235 skeleton views, and 21830 edges (Figure 1 shows the first $400 \mathrm{~m}$ ). Most of the edges are added from neighboring nodes along the same trajectory, but a good portion come from loop closures and parallel trajectories (Figure 5).

View matching has clearly captured the major structural aspects of the trajectory, relative to open-loop VO. It closed the large loop from the beginning of the trajectory to the end, as well as two smaller loops in between. We also measured the planarity of the trajectory: for the view-based system, RMS error was $22 \mathrm{~cm}$; for open-loop VO, it was $50 \mathrm{~cm}$.

Note that the vocabulary tree prefilter makes no distinction between reference views that are temporally near or far from the current view: all reference views are treated as places to be recognized. By exploiting the power of geometric consistency, there is no need to compute complex covariance gating information for data association, as is typically done for EKF-based systems [9, 10, 29, 32].

The time spent in view integration is broken down by

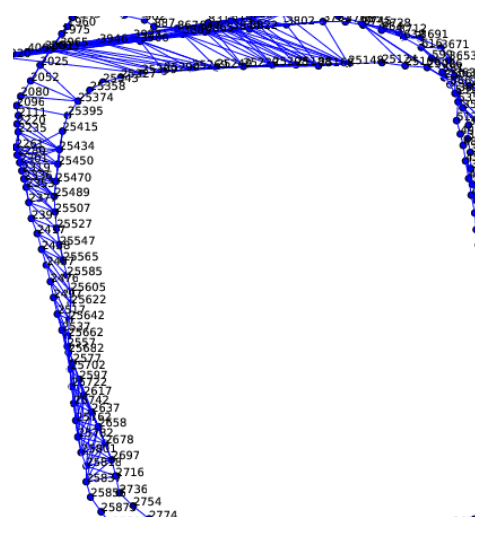

Fig. 5. A closeup from the office dataset showing the matched views on a small loop. The optimizer has been turned off to show the links more clearly.

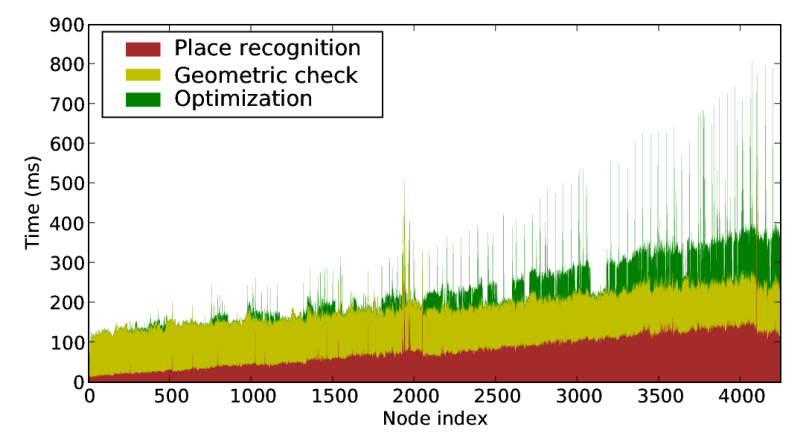

Fig. 6. Timing for view integration per view during the office loop trajectory.

category in Figure 6. The vocab tree prefilter grows linearly, to about $100 \mathrm{~ms}$ at the end; the geometry check is constant at $65 \mathrm{~ms}$. Toro does almost no work at the beginning of the trajectory, then grows to average $120 \mathrm{~ms}$ at the end, with maximum time of $500 \mathrm{~ms}$. VO can run at frame rates, while simultaneously adding and optimizing skeleton frames at $2 \mathrm{~Hz}$.

\section{B. Versailles Rond}

We ran viewmap on an outdoor urban sequence from a car in Versailles, a trajectory of about $400 \mathrm{~m}$ (Figure 7). The skeleton map contained 140 views, and PR found 12 matches after looping around, even when the car moved into an adjacent lane. The Versailles images have a lot of self-similarity in the windows, but the geometric check rejects false positives. This sequence easily runs online.

\section{Rough-Terrain Loops}

Large off-road trajectories present the hardest challenge for VSLAM. Grass, trees and other natural terrain have selfsimilar texture and few distinguishing landmarks. The dataset we used was taken by a very aggressive offroad autonomous vehicle, with typical motion of $0.5 \mathrm{~m}$ between frames, and sometimes abrupt roll, pitch, and vertical movement. VO fails on about $2 \%$ of the frames, mostly because of complete occlusion of one camera; we fill in with IMU data. There are two $5 \mathrm{~km}$ trajectories of $30 \mathrm{~K}$ frames that overlap occasionally. To test the system, we set the skeleton view distance to only 

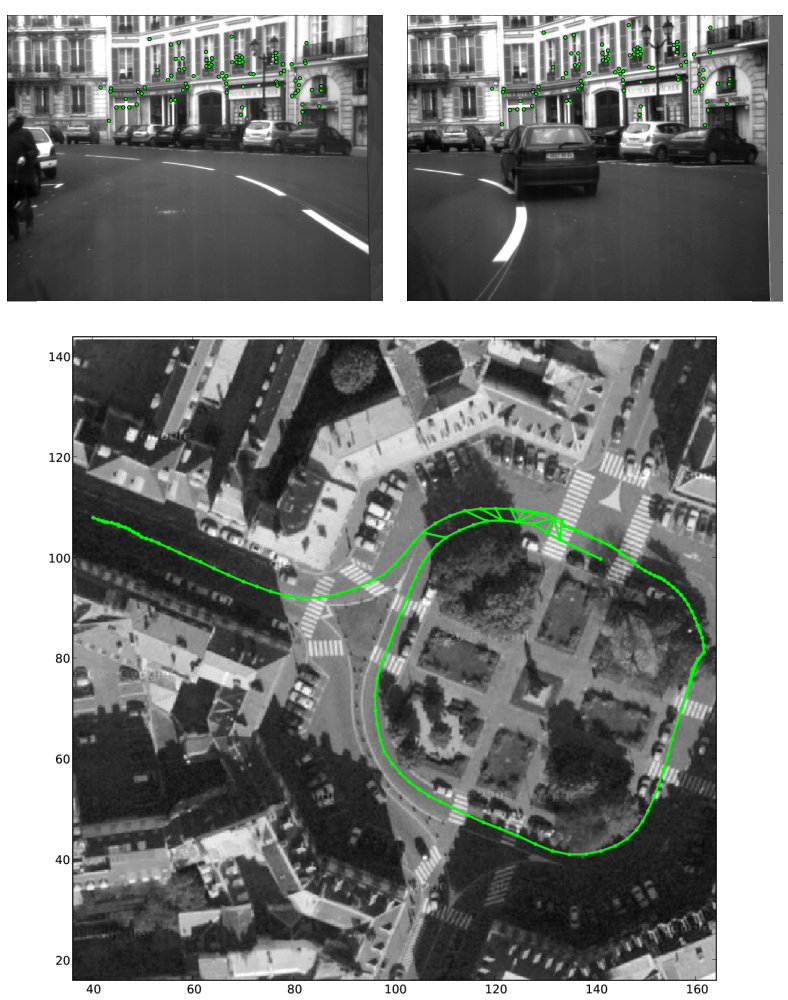

Fig. 7. Versailles Rond sequence of 700 video frames taken from a moving vehicle, $1 \mathrm{~m}$ baseline, narrow FOV. (Dataset courtesy of Andrew Comport [7]) Top: matched loop closure frames. Bottom: top-down view of trajectory superimposed on satellite image.
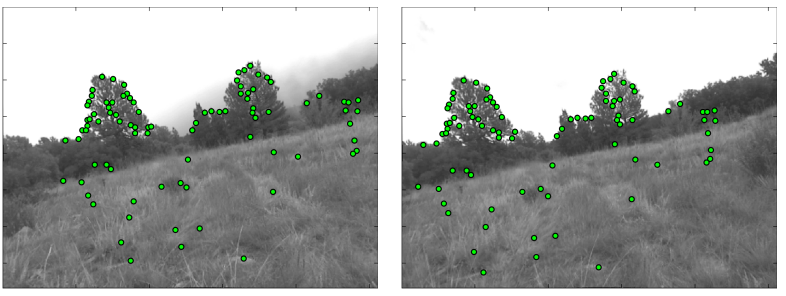

Fig. 8. Matched loop closure frames from the rough-terrain dataset. The match was made between two separate autonomous $5 \mathrm{~km}$ runs, several hours apart: note the low cloud in the left image.

$1 \mathrm{~m}$. The resultant graph has 14649 nodes and 69545 edges, of which 189 are cross-links between the two trajectories. The trajectories are largely corrected via the crosslinks - the error at the end of the loop changes from over $100 \mathrm{~m}$ with raw VO to less than $10 \mathrm{~m}$. Note that there are no loop closures within each trajectory, only between them. Figure 8 shows such a match. The PR system has the sensitivity to detect close possibilities, and the geometric check eliminates false positives - in Section $\mathrm{V}-\mathrm{C}$ we tested $400 \mathrm{~K}$ random image matches from this dataset, and found none with over 10 inliers (Figure 4).

\section{TrajectorySynth}

To showcase the capability of view integration, we performed a reconstruction experiment without any temporal information provided by video sequencing or $\mathrm{VO}$, relying just on view integration. We take a small portion of the office loop,

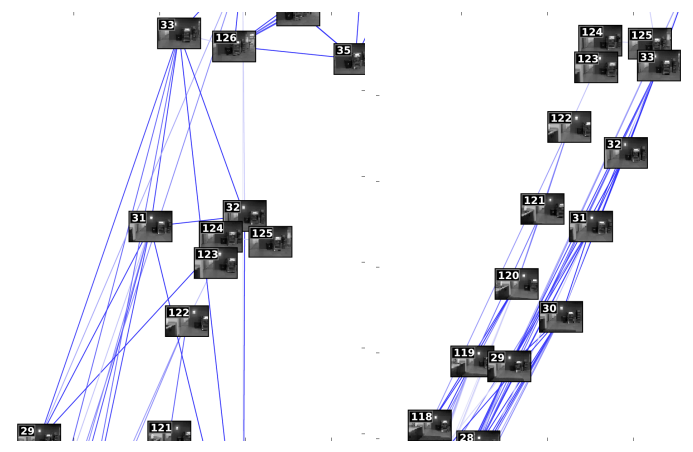

Fig. 9. Trajectory synthesis with no sequencing information: view constraints from PR at left; final optimized map at right.

extract 180 keyframes, and push them into the vocabulary tree. We then choose one keyframe as the seed, and use view integration to add all valid view matches to the view skeleton. The seed is marked as used, and one of the keyframes added to the skeleton is chosen as the next seed. The process repeats until all keyframes are marked as used.

The resultant graph is shown in Figure 9, left. The nodes are placed according to the first constraint found; some of these constraints are long-range and weak, and so the graph is distorted. Optimizing using Toro produces the consistent graph on the right. The time per keyframe is $150 \mathrm{~ms}$, so that the whole trajectory is reconstructed in 37 seconds, about 2 times faster than realtime. The connection to view stitching [31] is obvious, to the point where we both use the same term "skeleton" for a subset of the views. However, their method is a batch process that uses full bundle adjustment over a reduced set of views, whereas our approximate method retains just pairwise constraints between views.

\section{E. Relocalization}

Under many conditions, VO can lose its connection to the previous keyframe. If this condition persists (say the camera is covered for a time), then it may move an arbitrary distance before it resumes. The scenario is sometimes referred to as the "kidnapped robot" problem. View-based maps solve this problem with no additional machinery. To illustrate, we took the small loop sequence from the TrajectorySynth experiment, and cut out enough frames to give a $5 \mathrm{~m}$ jump in the actual position of the robot. Then we started the VO process again, using a very weak link to the previous node so that we could continue using the same skeleton graph. After a few keyframes, the view integration process finds the correct match, and the new trajectory is inserted in the correct place in the growing map (Figure 10). This example clearly indicates the power of constant re-recognition.

\section{F. Accuracy of View-Based Maps}

To verify the accuracy of the view-based map, we acquired a sequence of video frames that are individually tagged by "ground truth" 3D locations recorded by the IMPULSE Motion Capture System from PhaseSpace Inc. The trajectory is about $23 \mathrm{~m}$ in total length, consisting of 4 horizontal loops 

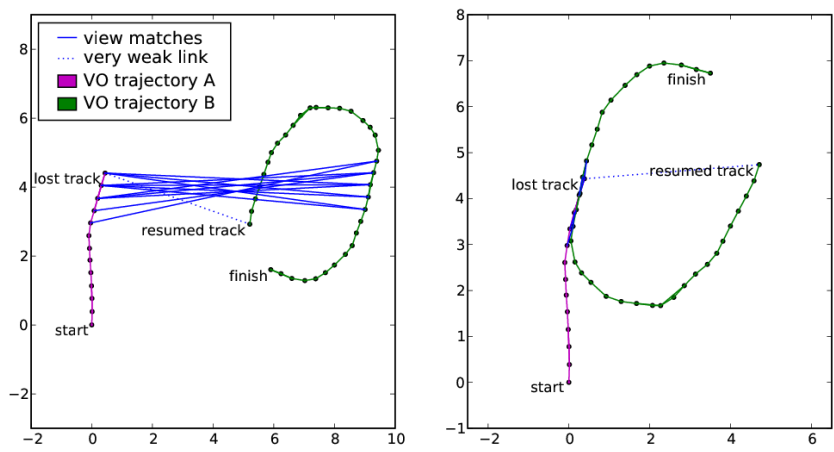

Fig. 10. Kidnapped robot problem. There is a cut in the VO process at the last frame in the left trajectory, and the robot is transported $5 \mathrm{~m}$. After continuing a short time, a correct view match inserts the new trajectory into the map.

with diameters of roughly $1.5 \mathrm{~m}$ and elevations from 0 to $1 \mathrm{~m}$. There are total of $6 \mathrm{~K}$ stereo images in the trajectory, with 224 graph nodes, and 360 edges. The RMS error of the nodes was $3.2 \mathrm{~cm}$ for the view-based system, which is comparable to the observed error for the mocap system. By contrast, open-loop VO had an error of $14 \mathrm{~cm}$.

\section{CONCLUSION}

We have presented a complete system for online generation of view-based maps. The use of re-recognition, where the robot's position is re-localized at each cycle with no prior information, leads to robust performance, including automatic relocalization and map stitching.

There are some issues that emerged in performing this research that bear further scrutiny. First, SGD optimization takes too long on very large graphs, since its convergence is sublinear. A better strategy is to use a few iterations of SGD, followed by Gauss-Seidel iterations to converge quickly. Second, we would like to investigate the monocular case, where full 6DOF constraints are not present in the skeleton graph.

\section{REFERENCES}

[1] M. Agrawal and K. Konolige. CenSurE: Center surround extremas for realtime feature detection and matching. In $E C C V, 2008$.

[2] M. Agrawal and K. Konolige. FrameSLAM: From bundle adjustment to real-time visual mapping. IEEE Transactions on Robotics, 24(5), October 2008

[3] O. Boiman, E. Shechtman, and M. Irani. In defense of nearest-neighbor based image classification. In Proceedings of IEEE Conference on Computer Vision and Pattern Recognition. IEEE, 2008.

[4] J. Callmer, K. Granström, J. Nieto, and F. Ramos. Tree of words for visual loop closure detection in urban slam. In Proceedings of the 2008 Australasian Conference on Robotics and Automation, page 8, 2008.

[5] M. Calonder, V. Lepetit, and P. Fua. Keypoint signatures for fast learning and recognition. In $E C C V, 2008$.

[6] M. Calonder, V. Lepetit, K. Konolige, P. Mihelich, and P. Fua. Highspeed keypoint description and matching using dense signatures. In Under review, 2009.

[7] A. Comport, E. Malis, and P. Rives. Accurate quadrifocal tracking for robust 3d visual odometry. In ICRA, 2007.

[8] M. Cummins and P. M. Newman. Probabilistic appearance based navigation and loop closing. In ICRA, 2007.
[9] A. Davison. Real-time simultaneaous localisation and mapping with a single camera. In ICCV, pages 1403-1410, 2003.

[10] A. J. Davison, I. D. Reid, N. D. Molton, and O. Stasse. Monoslam: Real-time single camera slam. IEEE PAMI, 29(6), 2007.

[11] E. Eade and T. Drummond. Monocular SLAM as a graph of coalesced observations. In Proc. ICCV, 2007.

[12] E. Eade and T. Drummond. Unified loop closing and recovery for real time monocular slam. In $B M V C, 2008$.

[13] F. Fraundorfer, C. Engels, and D. Nistér. Topological mapping, localization and navigation using image collections. In IROS, pages 3872-3877, 2007.

[14] U. Frese, P. Larsson, and T. Duckett. A multilevel relaxation algorithm for simultaneous localisation and mapping. IEEE Transactions on Robotics, 21(2):1-12, 2005

[15] G. Grisetti, D. L. Rizzini, C. Stachniss, E. Olson, and W. Burgard Online constraint network optimization for efficient maximum likelihood mapping. In ICRA, 2008.

[16] J. Gutmann and K. Konolige. Incremental mapping of large cyclic environments. In Proc. IEEE International Symposium on Computational Intelligence in Robotics and Automation (CIRA), pages 318-325, Monterey, California, November 1999.

[17] H. Jegou, M. Douze, and C. Schmid. Hamming embedding and weak geometric consistency for large scale image search. In ECCV, 2008.

[18] H. Jegou, H. Harzallah, and C. Schmid. A contextual dissimilarity measure for accurate and efficient image search. Computer Vision and Pattern Recognition, IEEE Computer Society Conference on, 0:1-8, 2007.

[19] A. Kelly and R. Unnikrishnan. Efficient construction of globally consistent ladar maps using pose network topology and nonlinear programming. In Proceedings 11th International Symposium of Robotics Research, 2003

[20] G. Klein and D. Murray. Parallel tracking and mapping for small AR workspaces. In Proc. Sixth IEEE and ACM International Symposium on Mixed and Augmented Reality (ISMAR'07), Nara, Japan, November 2007.

[21] G. Klein and D. Murray. Improving the agility of keyframe-based slam. In $E C C V, 2008$

[22] M. Klopschitz, C. Zach, A. Irschara, and D. Schmalstieg. Generalized detection and merging of loop closures for video sequences. In $3 D P V T$, 2008.

[23] K. Konolige and M. Agrawal. Frame-frame matching for realtime consistent visual mapping. In Proc. International Conference on Robotics and Automation (ICRA), 2007.

[24] K. Konolige, M. Agrawal, and J. Solà. Large scale visual odometry for rough terrain. In Proc. International Symposium on Research in Robotics (ISRR), November 2007.

[25] F. Lu and E. Milios. Globally consistent range scan alignment for environment mapping. Autonomous Robots, 4:333-349, 1997.

[26] M. Muja and D. Lowe. Fast approximate nearest neighbors with automatic algorithm configuration. In VISAPP, 2009.

[27] D. Nistér and H. Stewénius. Scalable recognition with a vocabulary tree. In $C V P R, 2006$.

[28] E. Olson, J. Leonard, and S. Teller. Fast iterative alignment of pose graphs with poor estimates. In In ICRA, 2006.

[29] L. Paz, J. Tardós, and J. Neira. Divide and conquer: EKF SLAM in O(n). IEEE Transactions on Robotics, 24(5), October 2008.

[30] J. Sivic and A. Zisserman. Video google: A text retrieval approach to object matching in videos. Computer Vision, IEEE International Conference on, 2:1470, 2003.

[31] N. Snavely, S. M. Seitz, and R. Szeliski. Skeletal sets for efficient structure from motion. In Proc. Computer Vision and Pattern Recognition, 2008.

[32] J. Solà, M. Devy, A. Monin, and T. Lemaire. Undelayed initialization in bearing only slam. In ICRA, 2005.

[33] B. Steder, G. Grisetti, C. Stachniss, S. Grzonka, A. Rottmann, and W. Burgard. Learning maps in $3 \mathrm{~d}$ using attitude and noisy vision sensors. In IEEE International Conference on Intelligent Robots and Systems (IROS), 2007.

[34] R. Unnikrishnan and A. Kelly. A constrained optimization approach to globally consistent mapping. In Proceedings International Conference on Robotics and Systems (IROS), 2002.

[35] B. Williams, G. Klein, and I. Reid. Real-time slam relocalisation. In ICCV, 2007 